\title{
Fetal Doppler Velocimetry
}

National Cancer Institute

\section{Source}

National Cancer Institute. Fetal Doppler Velocimetry. NCI Thesaurus. Code C114080.

An antenatal ultrasonog raphic method that measures blood flow velocity through either maternal or fetal vessels. 